\title{
Results of Spinal Fixation in Thoracolumbar Spine fractures with Spinal Cord Injury using the ASIA Impairement Scale
}

\author{
Authors \\ Dr Arnab Sinha1, Dr Amit Kumar ${ }^{1}$, Dr Santosh Kumar ${ }^{2}$ \\ ${ }^{1}$ Senior Resident, ${ }^{2}$ Additional Professor \\ Department of Orthopaedics, Indira Gandhi Institute of Medical Sciences, Patna 800001 Bihar \\ Corresponding Author \\ Dr Arnab Sinha \\ 302, Vishwanath Plaza, Near Dayanand High School, Mithapur, Patna 800001 Bihar
}

\begin{abstract}
Spinal fixation provides stability to the vertebral column but the treatment of unstable fractures of thoracolumbar spine has long been controversial. Probably the best way is to understand that Vertebral column disruption with neurodeficit (signifying spinal cord injury) means there is spinal instability as it denotes a failure of the stabilizing role of the vertebral column. Neurological status in complete and incomplete lesions was compared as per ASIA Impairement Scale after 6 months of surgery. An effort was done to find the effect of spinal fixation in different types of vertebral and cord lesions in our scenario. On the analysis of our results we found out that most of the patients presented after 24 hours of injury and that there were a larger percentage of complete spinal cord lesions than incomplete spinal cord lesions. The postfixation neurorecovery results by a comparison of the ASIA Impairement Scale preoperative and six months postoperative showed that there was neither sensory nor motor recovery in any of the patients of complete lesion whereas there was variable amount of motor and sensory recovery in incomplete lesions. We had our share of complications too. There were three cases of superficial infections, (which resolved with medication and dressings uneventfully), two of peroperative dural tear (controlled uneventfully) and two cases of implant breakage. Spinal fixation, fusion and decompression may help in early neurological recovery in incomplete lesion. Even a small amount of recovery in any lesion is very useful to the patient. Even in cases where there is no neurorecovery, stabilization of the spine by spinal fixation helps in early mobilization in both incomplete as well as complete lesions which is necessary for physical and occupational rehabilitation. Proper patient selection, meticulous surgery and a knowledge of prognosis is important and goes a long way in patient as well as doctor satisfaction. A review of the available relevant literature was also done.

Keywords- thoracolumber fractures, spinal cord injury, neurorecovery, ASIA Impairement Scale
\end{abstract}

\section{Introduction}

Archeological records from over 45,000 years ago developments in the field of spinal surgery and the are noted to describe Spinal Cord Injury (SCI) as "a disease one cannot treat" and that paralysis is management of SCI, the paralysis still remains incurable

incurable. Even after many remarkable 
Treatment of SCI is broadly divided into a) Care for Injured Cord b) Care for Injured Vertebrae c) Basic Care of the paralysed body.

Spinal Cord behaves like Central Nervous System. It resists any healing process or repair. Till date other than in experimental animal no surgery in human being has shown any evidence of regeneration or repair of the cord.

The treatment of vertebral fractures was traditionally conservative implying recumbency for 10 to 12 weeks followed by orthosis and gradual physiotherapy. Though literature is itself divided on the issue of conversion of conservative management to surgical management e.g. of fixation and stabilization but due to the availability of better implants and safe anaesthesia there is an increasing trend for early stabilization of unstable spine. Vertebral injury can be graded into four groups. Group- I Compression Fracture without neurological lesion but there is risk of deformity - kyphosis. This Can be treated with Bed rest and good Orthosis till bone heals. Fixation to prevent possible deformity is Surgeons' personal choice. Grade II-unstable Injury to Vertebral column with risk of neurological deterioration. Stabilization is indicated. Grade III- Mechanical and neurological failure leading to incomplete lesion.Stabilization and decompression is indicated. Grade IVMechanical and neurological failure leading to complete lesion or transaction. Reduction and stabilization is indicated.

Much emphasis is always put on neurological recovery to justify or contradict interventions in SCI, but even without neurological recovery, spinal fixation provides some positive non neurological advantages e.g. lesser complications, short length of stay, early mobilization and reduction in total cost of hospitalization and ease with basic care of the paralysed body

\section{Aim}

Neurorecovery is often advocated as the goal of spinal stabilization surgery in thoracolumber fractures with neurodeficit. The aim of this study was to find out the amount of neurorecovery in incomplete as well as complete spinal cord injuries using the ASIA Impairement Scale.

\section{Material and Methods}

Spinal fixation provides stability to the vertebral column but the treatment of unstable fractures of thoracolumbar spine has long been controversial. The concept of spinal stability is central to the field of spine surgery but, it is variably defined, widely interpreted, and inconsistently measured. Various scoring systems are in use but neurologic considerations are likely undervalued in the checklist scoring method. Probably the best way is to understand that Vertebral column disruption with neurodeficit (signifying spinal cord injury) means there is spinal instability as it denotes a failure of the stabilizing role of the vertebral column.

Keeping in view with these facts a retrospective study was done in thoraco-lumbar injury patients in whom spinal fixation along with decompression of the cord was performed. The inclusion and exclusion criteria of patients considered for the study were: Inclusion Criteria-Thoracolumbar Fractures presenting with neurodeficit (ASIA A,B,C and D). Exclusion Criteria-1.Cervical or Sacral fractures, 2.ASIA E patients, 3.Medically unstable patients, 4.polytrauma patients, 5. Patients refusing surgery. High dose methylprednesolone was administered to all patients presenting before 24 hours of injury at the standard dose of bolus $30 \mathrm{mg} / \mathrm{kg}$ then infusion $5 \mathrm{mg} / \mathrm{kg} / \mathrm{hr}$ for 24 hours.

Neurological status in complete and incomplete lesions was compared as per ASIA Impairement Scale after 6 months of surgery. An effort was done to find the effect of spinal fixation in different types of vertebral and cord lesions in our scenario. A review of the available relevant literature was also done.

\section{Results}

On the analysis of our results we found out that most of the patients presented after 24 hours of 
injury and that there were a larger percentage of complete spinal cord lesions than incomplete spinal cord lesions. The postfixation neurorecovery results by a comparison of the ASIA Impairement Scale preoperative and six months postoperative showed that there was neither sensory nor motor recovery in any of the patients of complete lesion whereas there was variable amount of motor and sensory recovery in incomplete lesions. We had our share of complications too. There were three cases of superficial infections, (which resolved with medication and dressings uneventfully), two of peroperative dural tear (controlled uneventfully) and two cases of implant breakage.

\begin{tabular}{|c|c|}
\hline Complete lesion & 32 \\
\hline Incomplete lesion & 24 \\
\hline Total & 56 \\
\hline $\begin{array}{l}\text { Presentation before } 24 \text { hours } \\
\text { of injury }\end{array}$ & 8 \\
\hline $\begin{array}{l}\text { Presentation after } 24 \text { hours of } \\
\text { injury }\end{array}$ & 48 \\
\hline $\begin{array}{l}\text { High dose methylprednesolone } \\
\text { was administered to all } \\
\text { patients presenting before } 24 \\
\text { hours of injury }\end{array}$ & $\begin{array}{l}\text { At a dose of bolus } \\
30 \mathrm{mg} / \mathrm{kg} \text { then } \\
\text { infusion } 5 \mathrm{mg} / \mathrm{kg} / \mathrm{hr} \\
\text { for } 24 \text { hours }\end{array}$ \\
\hline
\end{tabular}

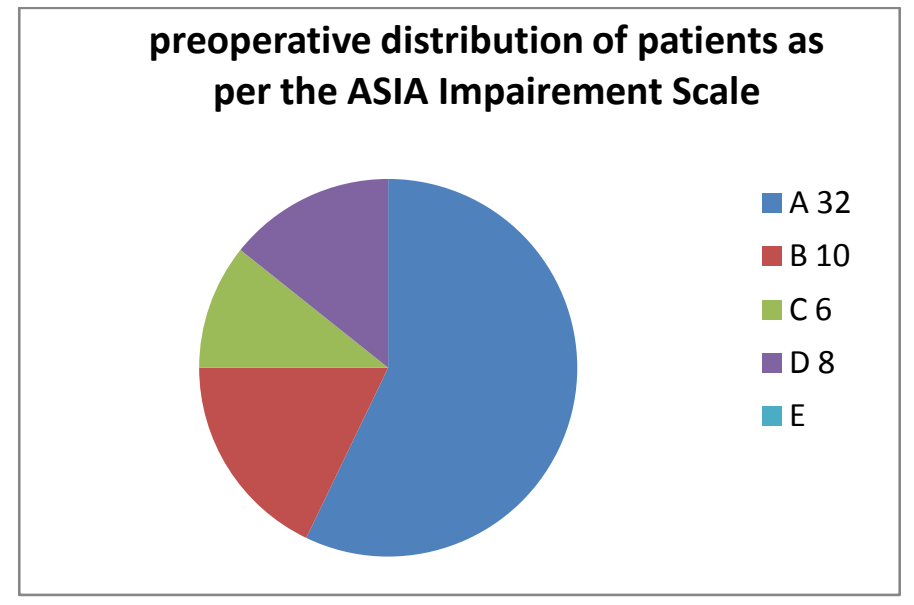

Neurological recovery as per the ASIA Impairement Scale at 6 months

\begin{tabular}{|l|l|}
\hline Complete lesion & 0 \\
\hline Sensory recovery & 0 \\
\hline Motor recovery & \\
\hline Incomplete lesion & Variable \\
\hline Sensory recovery & Variable \\
\hline Motor recovery & \\
\hline
\end{tabular}

Neurological status after six months of surgery as perASIA Impairement Scale
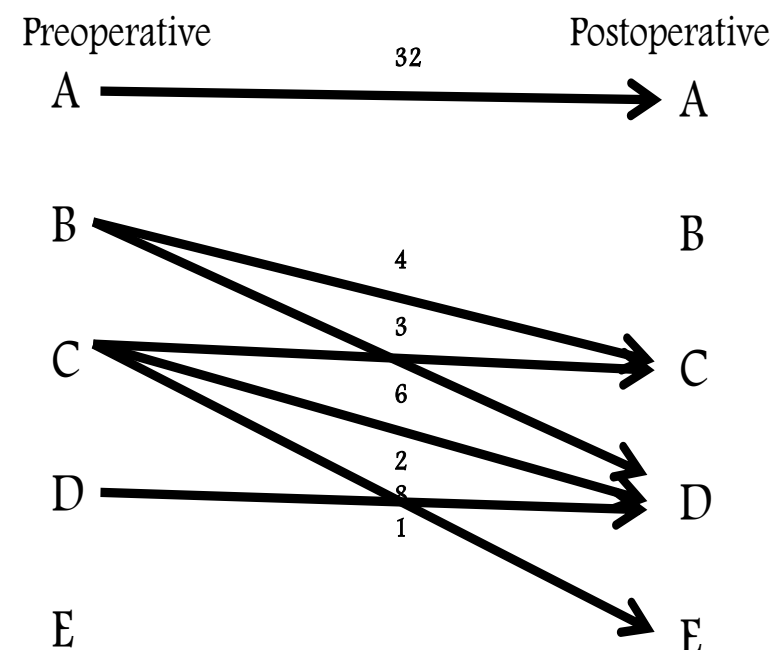

\begin{tabular}{|l|l|}
\hline Complications & 9 \\
\hline Infection & 3 \\
\hline Per operative dural tear & 2 \\
\hline Implant breakage & 2 \\
\hline
\end{tabular}

\section{Complications}

\section{Infection- 03}

Per operative duraltear-02

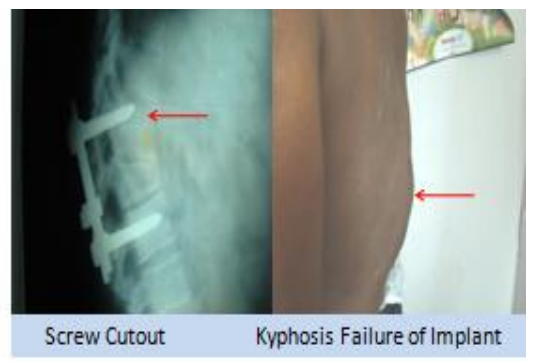

Implant breakage- 02

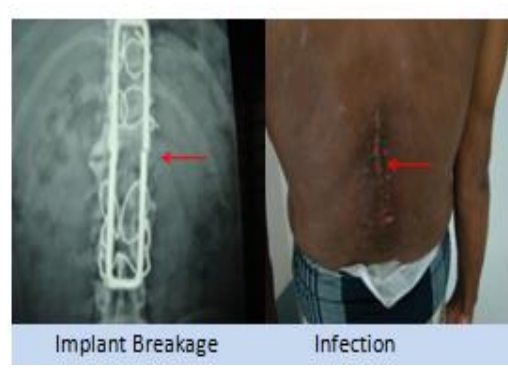

Page 9165 


\section{Discussion and review of literature}

Complete injuries don't recover. How much of neurorecovery of incomplete injuries can be attributed to the role of decompression and spinal fixation and how much to the natural recovery of the cord is questionable. Traditionally, opponents of surgery had an opinion that if surgical treatment is indicated for other reason, it should be addressed to that, but not addressed to clearing the canal or simple decompression and that neither decompression does not increase the chances of recovery. Nor does surgery lower the rate of complications and surgery has its own complication. Gradually with the development of safe anaesthesia and better implants surgery on the spine gained acceptance. The contemporary literature showed a positive tilt towards surgery. Provided that spine can be stabilized without further damage there should be little doubt about the benefits of early mobilization and ambulation of the patient. It improves psychological and social rehabilitation and there is less chronic pain. Also, neurological deterioration because of instability has been reported in two conservatively treated series. Much emphasis is always put on neurological recovery to justify or contradict interventions in SCI, but even without neurological recovery, spinal fixation provides some positive non neurological advantages e.g. lesser complications, short length of stay, early mobilization and reduction in total cost of hospitalization.

\section{Conclusion}

Spinal fixation, fusion and decompression may help in early neurological recovery in incomplete lesion. Even a small amount of recovery in any lesion is very useful to the patient. Even in cases where there is no neurorecovery, stabilization of the spine by spinal fixation helps in early mobilization in both incomplete as well as complete lesions which is necessary for physical and occupational rehabilitation. Proper patient selection, meticulous surgery and a knowledge of prognosis is important and goes a long way in patient as well as doctor satisfaction. No stabilization can prevent the complications of bad management of these neurotraumatised patients and surgery does not replace any of the components of good conservative care. Definitive conclusions should not be drawn regarding the prognosis for outcome and recovery after thoracolumber SCI. From a physiological standpoint, additional methodologically rigorous studies that take into consideration various levels of injury in more anatomically and physiologically relevant form are needed. Use of validated, comprehensive outcomes tools are important to improve our understanding of the impact of thoracolumber SCI and aid in examining factors in recovery from thoracic SCI.

ASIA Impairement Scale which was used preoperatively and on follow up at 6 months

A -Complete No motor or sensory function in the lowest sacral segment (S4 S5)

B- Incomplete Sensory function below neurologic level and in S4-S5,

no motor function below neurologic level

C- Incomplete Motor function is preserved below neurologic level

AND more than half of the key muscle groups below neurologic level have a muscle grade $<3$

D- Incomplete Motor function is preserved below neurologic level

AND at least half of the key muscle groups

below neurologic level have a muscle grade $=3$

E- Normal Sensory and motor function is normal

\section{References}

1. Hughes JT. The Edwin Smith Surgical Papyrus: an analysis of the first case reports of spinal cord injuries. Paraplegia 1988;26(2)

2. Bagnall et al. "Spinal fixation surgery for acute traumatic spinal cord injury (Review)" Cochrane Database of Systematic Reviews 2008, Issue 1 
3. Blackley HR, Plank LD, Robertson PA

Regeneration of spinal cord J Spinal Disorder 1996

4. Limb VK Grab Crisco JJet al Arch Phys Med Rehab 2006;75

5. American Academy Lecture 1998

6. Osti D, Harms J, Farey ID, Int.Orthop. 1997

7. Rabel KA, zorilla $P$ et al, Effects of decompression of cord on neurorecovery. Spine 1999; 24

8. Denis B,Holland NR et al Clin.Ortho. surgery vs nonoperaive treatment for unstable spine. 1999;289

9. Macevoy RV, Polk HC et al surgery for spinal stabilization Spine 1998,10

10. EpstainT, King HA et al Eur.spine J. 2007;32

11. Delphi convention 2002 time frame for surgical decompression afer spinal cord injury Spine 2011; 36

12. WilsonJR, et al Spinal Cord (2012)

13. McKinley W, et al, Arch Phys Med Rehab 2010

14. Vaccaro AR, Kim DH,Brodke DS. Diagnosis and management of thoracolumbar spine fractures JBJS 2011

15. Marshall et al. Deterioration following spinal cord injury A multicenter study Journal of Neurosurgery March 1987 / Vol. 66

16. Sinha A The rationale of spinal fixation in SCI - A Review of literature. Journal of Bihar Orthopaedic Association XXVI Oct 2015 vol 2

17. Sinha A, Singh NN Current Concepts Regarding Timing of Surgery and role of decompression in spinal fixation of spinal cord injury patients: A Review of literature. J Adv Med Dent Scie Res 2015;3(6):S88-S91 